\title{
INFLUENCE OF DIFFERENT TYPES OF ANESTHESIA FOR LAPAROSCOPIC HYSTERECTOMY ON THE DYNAMICS OF STRESS HORMONES
}

\author{
Ruslan Tkachenko \\ Department of obstetrics, gynecology \& reproductology \\ Shupyk National Medical Academy of Postgraduate Education \\ 9 Dorohozhytska str., Kyiv, Ukraine, 04112 \\ hexenal63@gmail.com \\ Stanislav Zaychenko \\ Department of anesthesiology \& intensive care \\ Kyiv City Center for Reproductive and Perinatal Medicine \\ 16 Heroiv Stalingradu ave., Kyiv, Ukraine, 04210 \\ zaychenkostanislav@gmail.com
}

\begin{abstract}
The aim of the study - to evaluate the influence of different variants of anesthesia, including low-opioid (LOA) and opioidfree anesthesia (OFA), on the dynamics of the stress response during laparoscopic hysterectomy.

Materials and methods: 102 patients were randomly allocated into 3 groups: 37 women assigned to receive a standard opioidbased anesthesia (OBA group), 33 women - LOA group and 32 patients OFA group.

Results: The patients of OFA group after induction and intubation of the trachea showed a significant $(p<0.05)$ increase in cortisol level by $155.4 \%$ and $160.9 \%$ compared with the OBA group and LOA group. After completion of the hysterectomy, regardless of the variant of anesthesia, the level of cortisol exceeded the preoperative one $\left(p_{\mathrm{OBA}}=0.116, p_{\mathrm{LOA}}=0.049, p_{\mathrm{OFA}}=0.043\right)$. Two hours after surgery with standard anesthesia (OBA group) there was a further increase in the concentration of cortisol in the blood, exceeding the initial values by $142.9 \%(p=0.043)$. Patients in the LOA group and OFA group tended to decrease cortisolemia. The changes in adrenaline \& glucose levels were somewhat similar.

Conclusion: Use of OFA is accompanied by an aggravation of the stress response at the stage of hysterectomy, which is manifested by an increase in the level of adrenaline and cortisol. The most adequate perioperative protection is inherent in low-opioid anesthesia, which is accompanied by a lower release of stress hormones during hysterectomy and in the postoperative period. Opioidbased anesthesia provides adequate intraoperative protection, but is accompanied by insufficient postoperative analgesia.
\end{abstract}

Keywords: opioid-free anesthesia, low-opioid anesthesia, stress hormones, laparoscopic hysterectomy.

DOI: $10.21303 / 2504-5679.2021 .001598$

\section{Introduction}

Hysterectomy is the most common major gynecological procedure in women [1], but hysterectomy for enlarged uteri is considered a challenge for gynecologic surgeons, due to the limit of exposure to surgical spaces [2]. Minimally invasive approaches as total laparoscopic hysterectomy should be used wherever possible [1] as it is the standard of care in majority of women diagnosed with endometrial cancer [3] and is feasible for enlarged uteri.

Despite the achievements of medicine and pharmacology, the problem of antinociceptive protection of patients from acute surgical pain remains unresolved in anesthesiology and surgery [4]. One of the main directions of development of modern anesthesiology is management of perioperative pain in order to limit the negative consequences of intra- and postoperative pain.

Opioids have been the «gold standard» of perioperative analgesia in most countries for many years. Traditionally, opioids have played a central role in balanced anesthesia, as they have helped control nociception and optimized hemodynamics [5], but have many side effects, such as respiratory depression, postoperative nausea and vomiting (PONB), itching, constipation, and so on. These side effects prolong the patient's hospital stay [6]. Opioid-induced respiratory depression is a major cause of opioid-related deaths [7]. Opioid side effects not only increase patient morbidity and poor perioperative course, but also increase the cost of care [8]. 
From 2015 to 2020, many publications appeared on new side effects of narcotic analgesics: opioid-induced postoperative hyperalgesia [9], opioid-induced postoperative immunosuppression and cancer progression [10,11], and opioid-induced reduction in the potency of local anesthetics [12].

Thus, opioid-related side effects and the problem of inadequate postoperative analgesia lead to the search for new methods of anesthesia without opioids or low-opioid anesthesia based on a multimodal approach to perioperative analgesia with non-opioid analgesics and adjuvants. It has been found that the use of OFA with nonsteroidal anti-inflammatory drugs, dexamethasone, lidocaine and ketamine either reduces or avoids intraoperative and postoperative use of opioids in patients after laparoscopic gynecological operations [13]. Dexmedetomidine has also shown adequate analgesia in patients undergoing laparoscopic surgery [14].

This study raises the relevance of the issue of finding an alternative to opioid anesthesia in laparoscopic hysterectomy, taking into account the problems of conducting a comprehensive audit study of the dynamics of stress hormones in the postoperative period in gynecological medicine with appropriate analysis of strategic approaches in the search for topical anesthesia.

The aim of the study - to evaluate the influence of different variants of anesthesia, including low-opioid (LOA) and opioid-free anesthesia (OFA), on the dynamics of the stress response during laparoscopic hysterectomy.

\section{Materials and methods}

This study is work performed on the base of the Kyiv City Center for Reproductive and Perinatal Medicine (Ukraine) during 2018-2020.

An open comparative method was used in the study in accordance with the requirements of the Pharmacological Committee of the Ministry of Health of Ukraine for limited clinical trials.

The study protocol was approved by the Local Ethical Committee by Shupyk National Medical Academy of Postgraduate Education No. 10 dated 20.11.2017 and the patients signed written fully informed consents in accordance with the Declaration of Helsinki.

All women assigned for hysterectomy were eligible for evaluation; the patients with ASA grade $>$ II, body mass index $(\mathrm{BMI})>35 \mathrm{~kg} / \mathrm{m}^{2}$, cardiac or chest diseases, previous open abdominal surgery, hypersensitivity to the drugs to be used were excluded from the study. All eligible women were clinically evaluated for demographic and baseline clinical data collection, underwent laboratory and radiological workup for assurance of inclusion and exclusion criteria.

102 patients were randomly allocated into 3 groups. The first control group included 37 women (aged 35-62, on average 47.4 \pm 10.3 year) assigned to receive a standard opioid-based anesthesia (OBA group), the second group included 33 women (aged 37-59, on average 46.6 \pm 7.4 year) assigned to receive low-opioids anesthesia (LOA group) and the third group included 32 patients (aged 38-60, on average 47.6 \pm 8.1 year) assigned to receive opioids-free anesthesia (OFA group).

Anesthesia protocol in the control (OBA) group: dexketoprofen $50 \mathrm{mg}$ IV to 10 minutes before surgery, induction - fentanyl $-2 \mathrm{mcg} / \mathrm{kg}+$ propofol $-1.5 \mathrm{mg} / \mathrm{kg}+$ atracuria besylate $-0.3 \mathrm{mg} / \mathrm{kg}$, followed by tracheal intubation and artificial ventilation of the lungs (AVL). During surgery, ventilation was controlled with a tidal volume of $6-8 \mathrm{ml} / \mathrm{kg}$, and the ventilatory rate was adjusted to maintain $\mathrm{EtCO}_{2}$ of 30-35 mm Hg. Patients were continuously non-invasively monitored for MAP and HR. Anesthesia was maintained by inhalation of sevoflurane $2.0-2.5 \mathrm{vol} \%$ until the MAC reached 0.9-1.0, and infusion of fentanyl 3-5 $\mathrm{mcg} / \mathrm{kg} / \mathrm{h}$.

Anesthesia protocol in the low-opiod (LOA) group: dexketoprofen $50 \mathrm{mg}$ IV to $10 \mathrm{~min}$ utes before surgery, induction - fentanyl $-2 \mathrm{mcg} / \mathrm{kg}+$ propofol $-1.5 \mathrm{mg} / \mathrm{kg}+$ atracuria besylate $0.3 \mathrm{mg} / \mathrm{kg}+$ ketamine $12.5 \mathrm{mg}$, followed by tracheal intubation and artificial ventilation of the lungs (AVL) with a tidal volume of $6-8 \mathrm{ml} / \mathrm{kg}$, and the ventilatory rate was adjusted to maintain $\mathrm{EtCO}_{2}$ of 30-35 mm Hg. Anesthesia was maintained by inhalation of sevoflurane $2.0-2.5 \mathrm{vol} \%$ until the MAC reached $0.9-1.0$, and infusion of ketamine $2-3 \mathrm{mkg} / \mathrm{kg} / \mathrm{min}+$ fentanyl $1.5-2 \mathrm{mcg} / \mathrm{kg} / \mathrm{h}+$ $+\mathrm{IV}$ paracetamol - $1000 \mathrm{mg}$ before the end of surgery.

Anesthesia protocol in the opiod-free (OFA) group: gabapentin $600 \mathrm{mg} 2$ hours before surgery; dexketoprofen $50 \mathrm{mg}$ IV to 10 minutes before surgery + lidocaine $1,0 \mathrm{mg} / \mathrm{kg}+$ dexmedetomidine $1.0 \mathrm{mkg} / \mathrm{kg}+$ dexamethasone $-8 \mathrm{mg}$. Induction - propofol - $1.5 \mathrm{mg} / \mathrm{kg}+$ atracuria besylate - 
$0.3 \mathrm{mg} / \mathrm{kg}+\mathrm{ketamine} 12.5 \mathrm{mg}$, followed by tracheal intubation and artificial ventilation of the lungs (AVL) with a tidal volume of $6-8 \mathrm{ml} / \mathrm{kg}$, and the ventilatory rate was adjusted to maintain $\mathrm{EtCO}_{2}$ of $30-35 \mathrm{~mm} \mathrm{Hg}$. Anesthesia was maintained by inhalation of sevoflurane $2.0-2.5$ vol \% until the MAC reached $0.9-1.0$, and infusion of ketamine $2-3 \mathrm{mkg} / \mathrm{kg} / \mathrm{min}+$ lidocaine $1.0 \mathrm{mg} / \mathrm{kg} / \mathrm{h}+$ + dexmedetomidine $0.6 \mathrm{mkg} / \mathrm{kg} / \mathrm{h}+\mathrm{IV}$ paracetamol - $1000 \mathrm{mg}$ before the end of surgery.

The hormonal homeostasis was studied by determination of cortisol concentration in the serum of women by enzyme-linked immunosorbent assay with the use of standard kits (Cortisol ELISA, DRG, Germany) and the level of adrenaline in the serum of patients - by high-performance liquid chromatography with an electrochemical detector. In addition, with the use of glucometer Finetest Auto-coding Premium (S. Korea) the dynamics of blood glucose level the was examined in an indicator of the impact of perioperative stress.

Studies were conducted at five phases: preoperative, after induction of anesthesia and tracheal intubation, traumatic phase of surgery (hysterectomy), 2 hours after the end of surgery, 24 hours after the end of surgery.

Statistical processing of the data obtained was carried out with the use of software package STATISTICA 10.0 (StatSoft, USA) and Primer of Biostatistics 4.03. Statistical analysis of the primary database conducted using the methods of parametric and nonparametric statistics. Checking the normality of the distribution of quantitative indicators conducted using the KolmogorovSmirnov criterion. Quantitative data with normal distribution are presented as the mean arithmetic and standard deviation $(\mathrm{M} \pm \mathrm{SD})$. For statistical analysis used the method of Kraskel-Wallis, Mann-Whitney U-test, Wilcoxon test. The value of $p<0.05$ was considered statistically significant.

\section{Results}

When comparing anthropometric indicators, we found no difference between the study groups by age, growth and body mass index (BMI) (Table 1).

Table 1

Distribution of patients by demographic and anthropometric indicators, M (SD)

\begin{tabular}{ccccc}
\hline Indicator & OBA $(\boldsymbol{n}=\mathbf{3 7})$ & LOA $(\boldsymbol{n}=\mathbf{3 3})$ & OFA $(\boldsymbol{n}=\mathbf{3 2})$ & $\boldsymbol{p}$ \\
\hline Age, years & $47.4(10.3)$ & $46.6(7.4)$ & $47.6(8.1)$ & 0.921 \\
Growth, cm & $163.8(5.8)$ & $164.4(4.9)$ & $164.1(6.3)$ & 0.932 \\
Weight, $\mathrm{kg}$ & $68.2(13.2)$ & $67.3(9.8)$ & $67.7(14.3)$ & 0.956 \\
BMI, $\mathrm{kg} / \mathrm{m}^{2}$ & $25.4(4.7)$ & $24.9(3.8)$ & $25.0(4.5)$ & 0.896
\end{tabular}

The formation of a stress response is always accompanied by an increase in the activity of the sympatho-adrenal and hypothalamic-pituitary-adrenal systems with an increase in the level of biologically active amines and hormones in the blood. We studied common surgical stress markers: cortisol, adrenaline, and blood glucose concentrations [15].

In the study of stress markers there was found, that preoperative serum concentrations of cortisol, adrenaline, and glucose were $203.9(158.0 ; 236.9) \mathrm{ng} / \mathrm{ml}, 414.4(287.4 ; 507.5) \mathrm{pmol} / \mathrm{l}$, and $4.60(4.00 ; 5.10) \mathrm{mmol} / 1$, respectively, and corresponded to the norm $(p=0.735 ; p=0.678$ and $p=0.593$, respectively), which indicated the absence of stress while awaiting surgery in patients of all groups.

The study of endocrine-metabolic changes that characterize the stress response showed, that the level of cortisol changed statistically significantly in the study stages within the groups, and depended on the variant of anesthesia $(p=0.038)$ (Table 2). Thus, patients of OFA group after induction and intubation of the trachea showed a significant $(p<0.05)$ increase in cortisol level by $155.4 \%$ and $160.9 \%$ compared with the OBA group and LOA group, which indicated an insufficient level of stress protection in the absence of opioids. After completion of the traumatic phase of surgery (hysterectomy), regardless of the variant of anesthesia, the level of cortisol exceeded the preoperative one $\left(p_{\mathrm{OBA}}=0.116\right.$, $\left.p_{\mathrm{LOA}}=0.049, p_{\mathrm{OFA}}=0.043\right)$ without statistical difference between the groups $(p=0.759)$. Two hours after surgery with standard anesthesia (OBA group) there was a further increase in the concentration of cortisol in the blood, exceeding the initial values by $142.9 \%(p=0.043)$, which indicated inadequate 
postoperative analgesia in these patients. Patients in the LOA group and OFA group tended to decrease cortisolemia. It was most significant in women of the OFA group, which indicated high-quality analgesia affected by multimodal analgesia. 24 hours after surgery cortisol levels decreased to preoperative ones and did not differ between the groups $(p=0.502)$.

The changes in adrenaline levels were somewhat similar (Table 3). Thus, patients of all groups after induction and intubation of the trachea showed a slight tendency to increase the level of adrenaline compared to its preoperative level, which can be explained by the relatively high preoperative levels of adrenaline due to psycho-emotional stress before surgery. After completion of the hysterectomy, regardless of the variant of anesthesia, the level of adrenaline exceeded the preoperative one $\left(p_{\mathrm{OBA}}=0.049, p_{\mathrm{LOA}}=0.119, p_{\mathrm{OFA}}=0.041\right)$ with statistical difference between the OBA group and OFA group in comparison with the LOA group, where the level of adrenaline was the lowest and did not differ statistically from the preoperative phase, which indicated the maximum stress protection in this group. Two hours after surgery with standard anesthesia (OBA group) there was a further increase in the concentration of adrenaline in the blood, exceeding the initial values by $32.2 \%(p=0.048)$, which indicated inadequate postoperative analgesia in these patients. Patients in the LOA group and OFA group tended to decrease adrenalinemia. It was most significant in women of the LOA group, which indicated high-quality analgesia affected by multimodal analgesia. 24 hours after surgery adrenaline levels decreased to preoperative ones, did not differ between the groups ( $p=0.602)$, and were significantly lower than in the previous phase.

Changes in glucose levels were similar, had no intergroup differences $(p=0.319)$, and did not depend on the variant of anesthesia $(p=0.499)$ (Table 4).

At the end of the main phase of surgery, regardless of the variant of anesthesia, the blood glucose level increased from the baseline one by $23.6 \%\left(p_{\mathrm{OBA}}=0.047\right), 19.8 \%\left(p_{\mathrm{LOA}}=0.076\right)$, and $24.4 \%\left(p_{\text {OFA }}=0.057\right) .2$ hours after surgery, patients in the OBA group had a further increase in blood glucose concentration, with its level exceeding the preoperative one by $39.6 \%(p=0.001)$. In patients of the OFA group and LOA group, glucose levels decreased and did not differ from the baseline ones ( $p=0.212$ and $p=0.155$, respectively). 24 hours after surgery, regardless of the variant of anesthesia used, the glycemic level decreased and did not differ significantly from the baseline one. At no stage of the study, the difference between the groups was statistically significant.

Table 2

Cortisol levels at research stages depending on the type of anesthesia $(\mathrm{M} \pm \mathrm{SD}, \mathrm{ng} / \mathrm{ml})$

\begin{tabular}{lccc}
\hline \multicolumn{1}{c}{ Phase } & \multicolumn{2}{c}{ Groups } & OFA \\
\cline { 2 - 4 } & OBA & LOA & $196.3 \pm 79.4$ \\
\hline Preoperative phase & $204.4 \pm 105.3$ & $210.5 \pm 99.1$ & $495.5 \pm 211.8 \#^{*}$ \\
After induction of anesthesia and tracheal intubation & $194.6 \pm 126.5$ & $189.9 \pm 80.7$ & $590.1 \pm 230.4 \#$ \\
Traumatic phase of surgery & $395.5 \pm 261.2^{*}$ & $408.9 \pm 220.1 \#^{*}$ & $297.7 \pm 153.3^{*}$ \\
2 hours after the end of surgery & $496.5 \pm 182.2 \#$ & $340.1 \pm 195.6 \#$ & $306.9 \pm 177.4$
\end{tabular}

Note: $\#-p<0.05$ compared with the preoperative phase; $*-p<0.05$ compared with the previous phase

Table 3

Adrenaline levels at research stages depending on the type of anesthesia $(\mathrm{M} \pm \mathrm{SD}, \mathrm{pmol} / \mathrm{l})$

\begin{tabular}{lccc}
\hline \multicolumn{1}{c}{ Phase } & \multicolumn{3}{c}{ Groups } \\
\cline { 2 - 4 } & OBA & LOA & OFA \\
\hline Preoperative phase & $504.4 \pm 105.3$ & $570.5 \pm 149.0$ & $506.3 \pm 177.5$ \\
After induction of anesthesia and tracheal intubation & $496.7 \pm 126.5$ & $529.5 \pm 180.7$ & $505.5 \pm 111.8$ \\
Traumatic phase of surgery & $676.5 \pm 169.6 \#^{*}$ & $608.9 \pm 120.1$ & $790.1 \pm 230.4 \#^{*}$ \\
2 hours after the end of surgery & $666.7 \pm 202.4 \#$ & $544.1 \pm 155.6$ & $597.7 \pm 143.7^{*}$ \\
24 hours after the end of surgery & $445.6 \pm 123.1^{*}$ & $490.7 \pm 120.2$ & $407.9 \pm 137.4^{*}$
\end{tabular}

Note: $\#-p<0.05$ compared with the preoperative phase; ${ }^{*}-p<0.05$ compared with the previous phase 
Table 4

Glucose levels at research stages depending on the type of anesthesia $(\mathrm{M} \pm \mathrm{SD}, \mathrm{mmol} / \mathrm{l})$

\begin{tabular}{lccc}
\hline \multicolumn{1}{c}{ Phase } & \multicolumn{3}{c}{ Groups } \\
\cline { 2 - 4 } & $4.71 \pm 0.52$ & LOA & OFA \\
\hline Preoperative phase & $4.52 \pm 0.70$ & $4.82 \pm 0.56$ & $4.84 \pm 0.67$ \\
After induction of anesthesia and tracheal intubation & $5.53 \pm 1.45^{*} \#$ & $5.39 \pm 1.26^{*}$ & $5.01 \pm 0.77$ \\
Traumatic phase of surgery & $6.12 \pm 1.89 \#$ & $5.43 \pm 1.02$ & $4.97 \pm 0.97$ \\
2 hours after the end of surgery & $5.02 \pm 1.21^{*}$ & $4.77 \pm 1.09$ & $4.31 \pm 0.65$
\end{tabular}

Note: $\#-p<0.05$ compared with the preoperative phase; $*-p<0.05$ compared with the previous phase

\section{Discussion}

Recently, enthusiasm for the benefits of opioid-free analgesia has been justified by many randomized trials [9, 16-18], which provide strong evidence of adequate postoperative analgesia compared to opioid-based anesthesia. But there are still many problems that still need to be addressed in this matter [19]. A multimodal approach is part of a method that helps minimize the use of opioids in the perioperative period, but limited research on the ideal combination of non-opioid excipients, their appropriate doses and stress-protective effects.

It is well known that surgical trauma causes a stress response in the body and is a complex cascade of neuroendocrine, metabolic, coagulation, inflammatory and immune responses that increase the body's ability to recover. This multilevel response to stress depends on a number of endogenous and exogenous factors, namely age, sex, previous health, type and invasiveness of surgery, type of anesthesia, pain intensity, etc. [20]. The pathogenic nature of the surgical stress response has been proven, which significantly worsens the postoperative period and prolongs the rehabilitation time of patients. Surgical stress response is the main cause of postoperative dysfunction of various organs and systems, which dictates the need to find approaches to its correction [21]. In this regard, limiting the severity of the surgical stress response becomes one of the leading tasks of anesthesia and postoperative intensive care [22]. The response to «stress» begins with the activation of the hypothalamic-pituitary-adrenal axis and the sympathetic nervous system. This leads to increased secretion of adrenocorticotropic hormone, cortisol, catecholamines and inflammatory mediators. The role of cortisol in stressful situations is to temporarily increase energy production by limiting processes that are not required for immediate survival [23].

Cortisol is one of the most commonly used markers of adaptive response (stress). Plasma cortisol concentrations may increase $2-10$-fold after induction of anesthesia, during surgery, and in the postoperative period and return to normal within 24 hours after surgery; however, depending on the severity of the surgical injury, it may remain elevated for 72 hours [24]. It is established that the degree of increase in the level of stress hormones in the blood is generally proportional to the severity of the injury. There is a clear relationship between surgical stress and cortisol, adrenaline and glucose levels in patients who have undergone surgery [23].

Adequate pain control cannot be achieved without the normalization of hormonal homeostasis, which is why it is very important to study the stress response of the patient's body using different anesthesia strategies during laparoscopic hysterectomy. This will help clarify the adequacy of stress protection for different anesthesia options.

As it turned out in our study, general anesthesia did not completely eliminate the stress response due to surgical trauma, because the hypothalamus and pituitary gland respond to harmful stimuli even with a deep level of anesthesia, but can limit it. This coincides with the data of I. Velickovic et al. [25]. The increase in the concentration of stress hormones in the serum during the operation occurred regardless of the anesthesia regimen. The most probable reason for this is the inability to provide absolute neurovegetative blockade with anesthetics used, and in patients with LOA and OFA group, possibly also due to the sympathomimetic action of ketamine.

The maximum increase against baseline cortisol, adrenaline and glucose levels in patients using standard OBA 2 hours after surgery is associated with the resumption of pain and activation 
of the sympathetic component of the autonomic nervous system due to the short duration of the analgesic effect of fentanyl and lack of preventive analgesia. This was confirmed by the formation of moderate and severe postoperative pain and the need for «rescue» analgesia in the first hours after surgery. Thus, when conducting standard OBA, there is a lack of post-anesthetic analgesia. The decrease in the concentration of stress markers occurred on the 1st day after surgery and was accompanied by a decrease in the intensity of pain, which indicated a sufficient level of analgesia. Against the background of combined intraoperative use a small doses fentanyl and sub-narcotic doses of ketamine combined with dexketoprofen \& paracetamol (LOA-group) at the end of the operation, a decrease a levels of cortisol, adrenaline and glucose was observed 2 hours after surgery, and after 24 hours their concentration reached baseline. This was accompanied by the absence of pain during the stages of the study, indicated adequate postoperative analgesia and proved the effectiveness of the applied anesthesia regimen.

The analgesic properties of sub-narcotic doses of ketamine have been confirmed in many clinical trials [26] and are due to the weakening of central sensitization caused by tissue damage and reduced development of tolerance to opioids. Nonsteroidal anti-inflammatory drugs do not have a direct effect on the classical stress response, but act on the synthesis of arachidonic acid metabolites, which are involved in various stages of the stress response [27], which requires further research in this direction.

Study limitations. A limitation of our study is to the level of stress response for second days of the postoperative period.

Prospects for further research. To investigate the influence of different variants of anesthesia in laproscopic hysterectomy not only on the indicators of stress response of the body, but also on hemodynamics, postoperative pain and psychological satisfaction of patients and postoperative cognitive dysfunction, which will allow to draw more weighty conclusions about the advantages and disadvantages of this variants of anesthesia.

\section{Conclusions}

1. The use of opioid-free multimodal anesthesia provides stress protection in the early postoperative period, but during the traumatic phase of surgery (hysterectomy) the level of stress protection is insufficient, accompanied by an increase in cortisol and adrenaline at this phase.

2. The most adequate perioperative protection is inherent in low-opioid anesthesia, which is accompanied by a lower release of stress hormones during hysterectomy and in the postoperative phase.

3. Opioid-based anesthesia provides adequate intraoperative protection, but is accompanied by insufficient postoperative analgesia.

\section{Conflict of interests}

The authors declare that they have no conflicts of interest.

\section{References}

[1] Obermair, A., Armfield, N. R., Graves, N., Gebski, V., Hanna, G. B., Coleman, M. G. et. al. (2019). How to train practising gynaecologists in total laparoscopic hysterectomy: protocol for the stepped-wedge IMAGINE trial. BMJ Open, 9 (5), e027155. doi: http://doi.org/10.1136/bmjopen-2018-027155

[2] Cianci, S., Gueli Alletti, S., Rumolo, V., Rosati, A., Rossitto, C., Cosentino, F. et. al. (2019). Total laparoscopic hysterectomy for enlarged uteri: factors associated with the rate of conversion to open surgery. Journal of Obstetrics and Gynaecology, 39 (6), 805-810. doi: http://doi.org/10.1080/01443615.2019.1575342

[3] Chambers, L. M., Carr, C., Freeman, L., Jernigan, A. M., Michener, C. M. (2019). Does surgical platform impact recurrence and survival? A study of utilization of multiport, single-port, and robotic-assisted laparoscopy in endometrial cancer surgery. American Journal of Obstetrics and Gynecology, 221 (3). doi: http://doi.org/10.1016/j.ajog.2019.04.038

[4] Ovechkin, A. M., Iavorovskii, A. G. (2019). Bezopioidnaia analgeziia v khirurgii: ot teorii k praktike. Moscow: GEOTARMedia, 240.

[5] Egan, T. D. (2019). Are opioids indispensable for general anaesthesia? British journal of anaesthesia, 122 (6), e127-e135. doi: http://doi.org/10.1016/j.bja.2019.02.018

[6] White, P. F. (2002). The Role of Non-Opioid Analgesic Techniques in the Management of Pain After Ambulatory Surgery. Anesthesia \& Analgesia, 94 (3), 577-585. doi: http://doi.org/10.1097/00000539-200203000-00019 
[7] Gupta, K., Nagappa, M., Prasad, A., Abrahamyan, L., Wong, J., Weingarten, T. N. et. al. (2018). Risk factors for opioid-induced respiratory depression in surgical patients: a systematic review and meta-analyzes. BMJ Open, 8, e024086. doi: http:// doi.org/10.1136/bmjopen-2018-024086

[8] Pizzi, L. T., Toner, R., Foley, K., Thomson, E., Chow, W., Kim, M. et. al. (2012). Relationship Between Potential Opioid-Related Adverse Effects and Hospital Length of Stay in Patients Receiving Opioids After Orthopedic Surgery. Pharmacotherapy: The Journal of Human Pharmacology and Drug Therapy, 32 (6), 502-514. doi: http://doi.org/10.1002/j.1875-9114.2012.01101.x

[9] Lavand'homme, P., Estebe, J.-P. (2018). Opioid-free anesthesia. Current Opinion in Anaesthesiology, 31 (5), 556-561. doi: http:// doi.org/10.1097/aco.0000000000000632

[10] Aich, A., Gupta, P., Gupta, K. (2016). Could Perioperative Opioid Use Increase the Risk of Cancer Progression and Metastases? International Anesthesiology Clinics, 54 (4), e1-e16. doi: http://doi.org/10.1097/aia.0000000000000112

[11] Lee, J. S.-J., Hu, H. M., Edelman, A. L., Brummett, C. M., Englesbe, M. J., Waljee, J. F. et. al. (2017). New Persistent Opioid Use Among Patients With Cancer After Curative-Intent Surgery. Journal of Clinical Oncology, 35 (36), 4042-4049. doi: http:// doi.org/10.1200/jco.2017.74.1363

[12] Liu, Q., Gold, M. S. (2016). Opioid-induced Loss of Local Anesthetic Potency in the Rat Sciatic Nerve. Anesthesiology, 125 (4), 755-764. doi: http://doi.org/10.1097/aln.0000000000001239

[13] Hakim, K. K., Wahba, W. B. (2019). Opioid-free total intravenous anesthesia improves postoperative quality of recovery after ambulatory gynecologic laparoscopy. Anesthesia: Essays and Researches, 13 (2), 199-203. doi: http://doi.org/10.4103/aer.aer_74_19

[14] Tufanogullari, B., White, P. F., Peixoto, M. P., Kianpour, D., Lacour, T., Griffin, J. et. al. (2008). Dexmedetomidine Infusion During Laparoscopic Bariatric Surgery: The Effect on Recovery Outcome Variables. Anesthesia \& Analgesia, 106 (6), 1741-1748. doi: http://doi.org/10.1213/ane.0b013e318172c47c

[15] Ezhevskaia, A. A., Prusakova, Zh. B. (2012). Clinical and biochemistry aspects of the endocrine-metabolic stress-response and hemostasic disorders in high intencity spinal surgery. Fundamentalnye issledovaniia, 4, 53-56.

[16] Kamdar, N. V., Hoftman, N., Rahman, S., Cannesson, M. (2017). Opioid-Free Analgesia in the Era of Enhanced Recovery After Surgery and the Surgical Home. Anesthesia Analgesia, 125 (4), 1089-1091. doi: http://doi.org/10.1213/ane.0000000000002122

[17] Boysen, P. G., Pappas, M. M., Evans, B. (2018). An Evidence-Based Opioid-Free Anesthetic Technique to Manage Perioperative and Periprocedural Pain. Ochsner Journal, 18 (2), 121-125. doi: http://doi.org/10.31486/toj.17.0072

[18] Beloeil, H. (2019). Opioid-free anesthesia. Best Practice \& Research Clinical Anaesthesiology, 33 (3), 353-360. doi: http:// doi.org/10.1016/j.bpa.2019.09.002

[19] Gupta, S., Mohta, A., Gottumukkala, V. (2020). Opioid-free anesthesia - caution for a one-size-fits-all approach. Perioperative Medicine, 9 (1). doi: http://doi.org/10.1186/s13741-020-00147-3

[20] Khalimonchyk, V., Klygunenko, O. (2019). Influence of components of anesthesia on dynamics of stress markers in gynecological laparoscopic surgery. Medical Perspectives, 24 (3), 33-39. doi: http://doi.org/10.26641/2307-0404.2019.3.181876

[21] Halabi, W. J., Jafari, M. D., Nguyen, V. Q., Carmichael, J. C., Mills, S., Stamos, M. J., Pigazzi, A. (2013). A Nationwide Analysis of the Use and Outcomes of Epidural Analgesia in Open Colorectal Surgery. Journal of Gastrointestinal Surgery, 17 (6), 1130-1137. doi: http://doi.org/10.1007/s11605-013-2195-4

[22] Nau, C. (2013). From preemptive to preventive analgesia. Anaesthesist, 62 (10), 787-788. doi: http://doi.org/10.1007/ s00101-013-2250-2

[23] Venkata Ramudu R. et al. (2015). Relationship between surgical stress and serum cortisol level: a comparative study among elective and emergencive surgery. Indo American Journal of Pharmaceutical Research, 5 (12), 3759-3764.

[24] Shaikh, S., Verma, H., Yadav, N., Jauhari, M., Bullangowda, J. (2012). Applications of Steroid in Clinical Practice: A Review. ISRN Anesthesiology, 2012, 1-11. doi: http://doi.org/10.5402/2012/985495

[25] Velickovic, I., Yan, J., Grass, J. A. (2002). Modifying the neuroendocrine stress response. Seminars in Anesthesia, Perioperative Medicine and Pain, 21 (1), 16-25. doi: http://doi.org/10.1053/sane.2002.30675

[26] Radvansky, B. M., Shah, K., Parikh, A., Sifonios, A. N., Le, V., Eloy, J. D. (2015). Role of Ketamine in Acute Postoperative Pain Management: A Narrative Review. BioMed Research International, 2015, 1-10. doi: http://doi.org/10.1155/2015/749837

[27] Doger, C., Aydin, G., Aksoy, M., Emre, C., Deveci, A., Bozkurt, M. et. al. (2014). The effect of anesthesia type on stress hormone response: Comparison of general versus epidural anesthesia. Nigerian Journal of Clinical Practice, 17 (4), $523-527$. doi: http://doi.org/10.4103/1119-3077.134058

Received date 25.11.2020

Accepted date 25.01.2021

Published date 29.01.2021
(C) The Author(s) 2021

This is an open access article under the CC BY license (http://creativecommons.org/licenses/by/4.0). 